\title{
Circular polarisation in the Crab nebula
}

from R. D. Davies

THE Crab nebula-the remnant of the supernova of AD 1054-is a very remarkable object. It is unique in that it has been detected over the entire electromagnetic spectrum from radio through infrared and the visual to $X$ rays and $\gamma$ rays. Moreover the pulsar with the shortest known period lies at its centre. This pulsar holds the key to the source of energy in the Crab nebula which had been a mystery before its discovery. The pulsar is a neutron star rotating at $30 \mathrm{~Hz}$. The important property of the pulsar in the context of the energy source of the Crab nebula is that it is slowing down - the rate of loss of rotational energy of the pulsar is equal to the energy radiated by the surrounding nebula.

The next stage is to understand the mechanism by which the pulsar supplies energy to the nebula. This energy is evident in the form of relativistic electrons and magnetic fields as these are required to produce the synchrotron emission from the nebula. But a simple estimate of the lifetime of the relativistic electrons which are responsible for the optical continuum emission indicates that they have a lifetime of only a few years. As a consequence such high energy electrons must be replenished continuously. This point was clearly recognised in the earliest work on the topic. Oort and Walraven in 1956 suggested that the moving wisps of gas seen near the centre of the nebula were evidence of continuing activity from a central star.

Recently Rees and Gunn (Mon. Not. $R$. astr. Soc., 167, 1; 1974) have looked afresh at the problem of the supply of relativistic electrons and magnetic fields to the nebula. They took a theory originally proposed by Piddington in 1957 and discussed subsequently by Kardeshev which had a central object responsible for winding up the magnetic field and thereby maintaining its strength. Rees and Gunn showed that the magnetic flux through the object increases in proportion to the number of ratations of the central pulsar; this indicates that as the nebula ages the magnetic field becomes increasingly important. The configuration of the field will be toroidal with an axis parallel to the spin axis of the pulsar. This axis is taken to lie along the major axis of the nebula (NW-SE). Linear polarisation measurements at optical and radio wavelengths show that the magnetic field is uniform and is essentially perpendicular to the major axis as would be expected from a toroidal configuration if the axis of rotation is in the plane of the sky.

Synchrotron theory indicates that more stringent tests can be made of the field configuration by measuring circular polarisation. Legg and Westfold (Astrophys. J., 154, 499; 1968) made a detailed analysis of the polarisation produced in the synchrotron mechanism and showed that a small component of circular polarisation is present. The sense of the circular emission depends on the inclination of the line of sight to the normal to the magnetic field, while the degree of polarisation is proportional to the square root of the magnetic field divided by the frequency. Such circular polarisation has already been detected in the radio emission from the magnetosphere of Jupiter and from several fiat spectrum (that is, compact) extragalactic radio sources. It is now of interest to see if circular polarisation can be detected in the Crab nebula in the configuration suggested by the Rees and Gunn toroidal field model. Optical measurements by Martin et al. (Mon. Not. R. Astr. Soc., 159, 191; 1972) showed the presence of a weak circular polarisation but it is believed that this is produced by dust in the interstellar medium lying between the Crab nebula and the Earth.

\section{Models of nitrogen fixation}

\section{from A.J. Thomson}

THE ability of primitive bacteria and some blue-green algae to reduce atmospheric nitrogen to ammonia at ambient temperature and pressure has long been a provocation to inorganic chemists. Until the mid-1960s, the only other known reaction of nitrogen at room temperature was the formation of metal nitrides either on clean metal surfaces or by lithium and alkaline earth metals. Considerable progress has been made in the isolation and analysis of the enzyme, nitrogenase, responsible for biological fixation (Hardy, Burns and Parshall, Adv. Chem. Ser., 100, 219; 1971). High purity preparations are now available from three genera of bacteria, including Azotobacter vinelandii and Clostridium pasteurianum. The enzyme, which requires an electron source such as ferredoxin or sodium dithionite, can be separated into two proteins, a high molecular weight component containing one or, less probably, two atoms of molybdenum and about 15 atoms of iron per molybdenum atom. The smaller protein contains two atoms of iron and two ions of 'labile' sulphide. Little is known about the environment of the metal atoms, although the high sulphur content of the protein
No similar effect is produced by dust at radio wavelengths. An interesting new measurement of significantly higher sensitivity than made previously is now forthcoming. Weiler ( this issue of Nature, page 24) reports the detection of circular polarisation in the radio emission from the Crab nebula at $1,415 \mathrm{MHz}$. Using the Westerbork Aperture Synthesis Radio telescope he finds a fractional polarisation of about $0.05 \%$ in four regions in the nebula. The circular polarisation is however of one sense only and does not show the change of sense in adjacent quadrants predicted on the toroidal model. Weiler concludes that the simplest magnetic field structure consistent with his observations is that of a relatively uniform field of $10^{-3}$ to $10^{-4}$ gauss with its line of sight component directed towards the Earth. On the face of it Weiler's detection is significantly above his measurement errors, although as he himself admits, independent confirmation would be useful at a lower frequency ( $\lesssim 1 \mathrm{GHz}$ ). If indeed this result is confirmed we are left with the need for a mechanism to maintain and amplify the magnetic field of the Crab nebula. has led to the suggestion that sulphur is a ligand. There is no direct evidence that the metal atoms are directly involved in binding nitrogen during fixation.

The discovery by Allen and Senoff (Chem. Commun., 221, 1965) of the first metal dinitrogen complex, $\left[\mathrm{Ru}\left(\mathrm{NH}_{3}\right)_{5}\left(\mathrm{~N}_{2}\right)\right]^{2+}$, during the attempted synthesis of $\left[\mathrm{Ru}\left(\mathrm{NH}_{3}\right)_{6}\right]^{2+}$ by the reaction of hydrazine with ruthenium trichloride trihydrate in water showed that $\mathrm{N}_{2}$ could be made to ligand to metals. This was an important step since it had been a puzzling feature of inorganic chemistry that, whereas so many carbon monoxide complexes of low-valent metals were known, the isoelectronic $\mathrm{N}_{2}$ molecule was apparently unable to bind. Since this observation some fifty complexes of dinitrogen have been reported (Sellman, Angew. Chem. Int. Edit., 13, 639; 1974). It is now a tacit assumption of all model studies that a metal ion, most probably molybdenum, is responsible for binding dinitrogen in nitrogenase.

The primary process of fixation, the trapping of the nitrogen molecule at ambient partial pressure, has now been successfully modelled by several groups using well defined compounds. A group 